\title{
Implementación de protecciones y simulación automática de eventos para localización de fallas en sistemas de distribución de energía
}

\author{
Juan J. Mora *\$, Juan C. Bedoya*，y Joaquim Meléndez** \\ * Programa de Ingeniería Eléctrica de la Universidad Tecnológica de Pereira (UTP) \\ ** Facultad de Ingenierías de la Universidad de Girona (UdG), España. \\ §e-mail:jjmora@utp.edu.co
}

(Recibido: Marzo 13 de 2006 - Aceptado: Julio 11 de 2006)

\begin{abstract}
Resumen
La localización de fallas en sistemas de distribución es de gran importancia para la calidad del servicio de energía eléctrica, especialmente en lo relacionado con la continuidad del suministro. Para localizar fallas se usan comúnmente dos tipos de métodos, los basados en el modelo y en el conocimiento. Los métodos basados en el conocimiento usan información de las señales de tensión y corriente registradas durante la falla, y normalmente requieren de gran cantidad de datos de fallas para desarrollar adecuadamente su función. Este artículo presenta la forma de modelar las protecciones de un sistema de distribución en un esquema de salvamento de fusibles y la obtención de una base de datos de fallas, con generación automática de eventos por medio del programa alternativo de transitorios (ATP) y Matlab. La base de datos obtenida puede ser utilizada para realizar diferentes tipos de análisis del sistema, y en este caso en particular, para resolver el problema de localización de fallas en sistemas de distribución usando métodos basados en el conocimiento. Como resultado se desarrolló una base de datos con 930 simulaciones de fallas diferentes, en un circuito de prueba de 25 nodos.
\end{abstract}

Palabras clave: Calidad de energía, Localización de fallas, Sistemas de distribución.

\begin{abstract}
Fault location in power distribution systems is an important aspect of power quality, in particular, in relation to power supply continuity. Currently, there are two clearly defined methods commonly used to locate faults, those which are based on the power system model and those which are based on knowledge. Fault location methods based on knowledge use information obtained from signals of voltage and current measured during fault situations, and normally require a considerable amount of fault registers to have a successful performance. This paper presents the modeling of a power distribution system and its protective relaying, settled in a fuse saving scheme. Using the described power system, it is possible to obtain a fault database by means of an event automatic generation using the Alternative Transient Program (ATP) and Matlab. The fault database can be used to perform different types of system analysis and in this specific case to solve the problem of fault location in power distribution systems using knowledge based methods. As a result, a data base which contains 930 different fault simulations on a 25 node test system was obtained.
\end{abstract}

Keywords: Power Quality, Fault location, Distribution systems. 


\section{Introducción}

La calidad de la energía se ha convertido en un tema de estudio de gran interés tanto para los operadores de red como para los usuarios. Entre los aspectos más importantes de la calidad se tienen en cuenta, la forma de onda y la continuidad del servicio. La actual importancia de estos aspectos radica en las políticas de regulación estipuladas por los entes de los diferentes países, que para el caso Colombiano es la Comisión Reguladora de Energía y Gas, CREG. En cuanto la continuidad del servicio existen dos indicadores dados por la CREG, y son: a) el índice DES (Duración Equivalente de las Interrupciones del Servicio), que mide el tiempo total durante el cual la energía no es suministrada; y b) el índice FES (Frecuencia Equivalente de las Interrupciones del Servicio), que mide el número total de interrupciones del servicio. La CREG ha establecido límites para estos indicadores que los operadores de red deben respetar so pena de pagar compensaciones por el incumplimiento de la reglamentación (Torres et al., 2001; CREG, 19982002 ).

La eficiente y oportuna localización de las fallas, ayudará a las empresas distribuidoras a mejorar sus índices DES y FES. La rápida localización de fallas consideradas como permanentes, permitirá disminuir la duración de la interrupción (DES), mientras que el continuo monitoreo y localización de las fallas permanentes y transitorias permitirá a las empresas determinar las debilidades de su sistema y de esa forma podrán fortalecer la red de modo que la ocurrencia de fallas (FES) será cada vez menor (Carrillo \& Ordóñez, 2002).

En este artículo se plantea la implementación de un circuito de distribución y sus protecciones, así como la simulación automática de fallas en ATP (Alternative Transient Program) mediante una interfase de instrucciones elaborada con el software Matlab®. Esta simulación permite obtener la base de datos con registros de fallas para entrenar y validar las metodologías basadas en el conocimiento utilizadas para localización de fallas.

La segunda sección de este artículo presenta el planteamiento del problema de localización de fallas en sistemas de distribución. En la sección 2.1 se presentan los fundamentos conceptuales para el desarrollo de métodos basados en el conocimiento para localización de fallas. A continuación, se presenta el modelado del sistema de distribución y elementos de protección en la sección 2.2. En el numeral 2.3 se presenta el procedimiento para la generación automática de fallas. En la sección 3 se presentan los resultados obtenidos y finalmente en la sección 4 se presentan las conclusiones de este trabajo.

\section{Metodologia}

\subsection{Localización de fallas en sistemas de distribución}

La localización de fallas en sistemas de distribución, es un problema muy complejo debido a que éstos presentan muchas ramificaciones y tienen pocos sistemas de medición dentro de la red. Para la solución de este problema se han utilizado diferentes metodologías que se pueden clasificar en dos grandes grupos: las que usan el modelo de la red y las que aprovechan otro tipo de información del sistema (Das, 1998).

Los métodos basados en el modelo (MBM), más comúnmente usados para localización de fallas, utilizan el valor RMS del fundamental de tensión y de corriente para estimar la impedancia vista desde la subestación del sistema de distribución hasta el lugar donde se encuentra localizada la falla. Esta impedancia es convertida a una distancia equivalente a partir de los parámetros de la red. Sin embargo, debido a la alta ramificación del sistema, existe una múltiple estimación de la sección que está bajo falla, y además presenta el inconveniente de la alta dependencia del modelo del sistema (Mora et al., 2005a).

De otra parte, los métodos que aprovechan otro tipo de información se conocen como basados en el conocimiento (MBC), y usan la señal en el tiempo de tensión y corriente, como el tipo de carga conectada en cada nodo, ubicación de las protecciones e información de las fallas ocurridas anteriormente en la red. Estos métodos son un excelente complemento de los MBM y permiten reducir el problema de la múltiple estimación del sitio de falla (Mora et al., 2005b). 
Para una eficiente implementación de los MBC, se necesita una gran cantidad de información de fallas ocurridas en los diferentes nodos del sistema. Normalmente, las empresas distribuidoras de energía no poseen esta información y por lo tanto se tiene que recurrir a las técnicas de simulación que permitan obtener una base de datos con registros de fallas. Una herramienta de simulación es el ATP, que es ampliamente utilizado a nivel mundial por los investigadores en el área de sistemas eléctricos de potencia y especialmente para el análisis de fallas y fenómenos transitorios. Una ventaja adicional es que el ATP es de libre uso para propósitos académicos y permite que el usuario pueda hacer aportes para su mejoramiento, lo que ha permitido su rápida evolución (ATP, 2002)

2.2 Desarrollo de un método basado en el conocimiento (MBC) para la localización de fallas

La implementación de un $\mathrm{MBC}$ para la localización de fallas requiere de un planteamiento metodológico, el análisis de técnicas con las cuales se puede implementar, así como la información que se utilizará para entrenamiento y validación de los MBC.

\subsubsection{Etapas metodológicas}

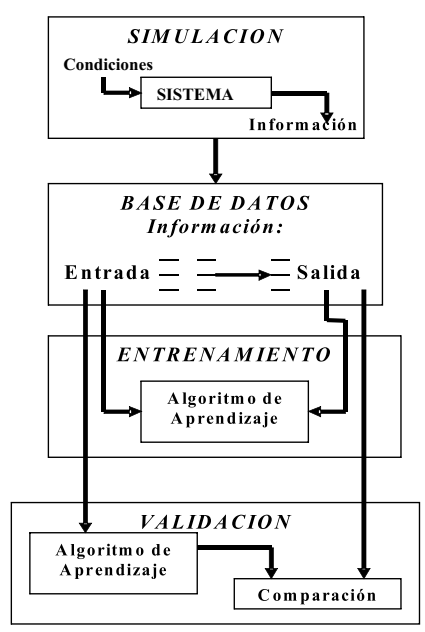

Figura 1. Esquema de las etapas metodológicas necesarias para construir una herramienta de modelado de los conocimientos extraídos de las señales de tensión y corriente y que permitan resolver el problema de localización de fallas.
La figura 1 muestra el esquema de las etapas necesarias para desarrollar una metodología que permita modelar los conocimientos extraídos de las señales de tensión y corriente y que permitan resolver el problema de localización de fallas.

La simulación permite adquirir las medidas que muestren el sistema bajo estado de falla. De allí se extrae información de las condiciones impuestas sobre el sistema y los resultados de interés, como las señales de tensión y corriente, entre otra información que se describe en el numeral 2.2.3. Se requiere luego, formar una base de datos en la que esté contenida la información del sistema bajo diferentes condiciones. Esta información se organiza en forma Entrada-Salida antes de entrar a la etapa de entrenamiento.

Existen diferentes algoritmos que pueden reproducir el conocimiento a partir de ejemplos. Para ello es necesario un aprendizaje ó entrenamiento que consiste en presentarle al algoritmo el conjunto de datos de entrada y salida organizados en la base de datos, para el caso que se requiera hacer un aprendizaje supervisado.

Finalmente, hay una etapa de validación en la que se busca comprobar la efectividad del MBC. El error se estima por la comparación entre el valor de salida del algoritmo ante una entrada específica y el valor de salida esperado.

\subsubsection{Técnicas comúnmente empleadas en $\operatorname{los} \mathrm{MBC}$}

Los MBC normalmente formulan la solución de un problema mediante un algoritmo de clasificación que usa un conjunto de datos de EntradaSalida cuyo número es suficientemente significativo. El objetivo es conseguir que el MBC aprenda automáticamente las propiedades deseadas a partir de estos ejemplos, mediante una modificación apropiada de sus parámetros. El proceso en el cual se adecúan dichos parámetros a la resolución de cada problema, se denomina aprendizaje ó entrenamiento.

Algunos de los MBC empleados comúnmente son: El método LAMDA (Learning Algorithm for Multivariate Data Analysis) que es un algoritmo 
de aprendizaje que permite el análisis de datos multivariables mediante una metodología de clasificación estadística (Waissman et al., 2000); las Redes Neuronales Artificiales (RNA) (Martins et al., 2003; Li-Xing, 1997); los Sistemas de Inferencia Difusa (FIS) que permiten codificar el conocimiento de un problema específico a través de reglas o etiquetas lingüísticas del tipo IFTHEN (Russell \& Norvig, 2002; Li-Xing, 1997); los Sistemas Adaptativos de Inferencia NeuroDifusa (ANFIS) incorporan las ventajas de las RNA y los FIS (Russell \& Norvig, 2002; Li-Xing, 1997); las Máquinas de Soporte Vectorial, (SVMs, Support Vector Machines), son una evolución de las RNA, y clasifican con un hiperplano óptimo de separación (OSH) que separa los datos de entrada y los cataloga en alguna de las clases establecidas (Russell \& Norvig, 2002; Burges, 1998); y las Redes Bayesianas, son un modelo probabilístico multivariado que relaciona un conjunto de variables aleatorias mediante un grafo dirigido (Cormane et al., 2005), entre otros.

\subsubsection{Información requerida}

Aunque la mayor parte de la información que describe al sistema de distribución bajo estado de falla, pertenece básicamente a los registros en el dominio del tiempo de tensión y corriente medidos en la subestación, existe información adicional que es útil para resolver el problema de localización de fallas. El registro de llamadas telefónicas hechas por los clientes informando sobre fallas en la red, la tipificación de la carga (tipo de carga que existe en cada nodo, industrial comercial y residencial, y para este último la estratificación social) y las condiciones climáticas, son ejemplos de este tipo de información.

La información que permite caracterizar el sistema bajo falla se conoce como descriptores, entre los cuales tenemos (Mora et al., 2005c):

A). Caracterización de los huecos de tensión: La magnitud RMS de la señal de tensión registrada en la subestación tiene una magnitud variable durante los intervalos de prefalla, falla y postfalla. La caracterización de huecos de tensión permiten extraer descriptores que se pueden asociar con las fallas tal como se presenta en (Mora, 2003).
B). Análisis de la señal transitoria: Las componentes de frecuencia y duración del transitorio, se pueden relacionar con la localización de la falla tal como se muestra en (Martínez \& Castro, 2002).

C). Análisis de coeficientes $\alpha$ y $\beta$ : Esta metodología se fundamenta en la transformación de las muestras de las tres señales de corriente de falla, utilizando la transformada de ClarkConcordia (Martins et al., 2003).

D). Análisis de los patrones de la corriente de carga: Esta metodología permite caracterizar los registros de conmutación de los elementos de protección del sistema de distribución. La actuación de reconectadores, seccionalizadores, interruptores en conjunto con relés de sobrecorriente instantáneos y de relación inversa tiempo corriente y fusibles, depende de su tiempo de calibración (Lewis, 1998; Seung-Jae et al., 2004).

\subsection{Modelado del sistema de distribución y elementos de protección}

La herramienta de simulación utilizada es el ATP, seleccionada por su gran aceptación en la comunidad científica del área. Es una herramienta de libre uso y permite obtener información de interés de las variables de un sistema de potencia, tanto en estado estable como en el estado transitorio en condiciones de falla.

La figura 2 muestra un sistema de pruebas de 25 kV SaskPower en Saskatoon, Canadá (Seung-Jae et al., 2004). Al sistema de distribución se han adicionado cinco elementos de protección que son: Un interruptor principal (52) junto con un relé de sobre corriente de relación inversa tiempo corriente (51) en el nodo de la subestación; un reconectador $(\mathrm{R})$ al inicio de la línea que une los nodos 6 y 7; un fusible en las línea que unen los nodos 9 y 14 (F1); otro fusible en la línea que une los nodos 10 y 21 (F2), y un último fusible en la línea que une los nodos 15 y 17 (F3). El sistema de protección fue modelado en el esquema de salvamento de fusibles (Seung-Jae et al., 2004; Phadke \& Horowitz, 1995). 


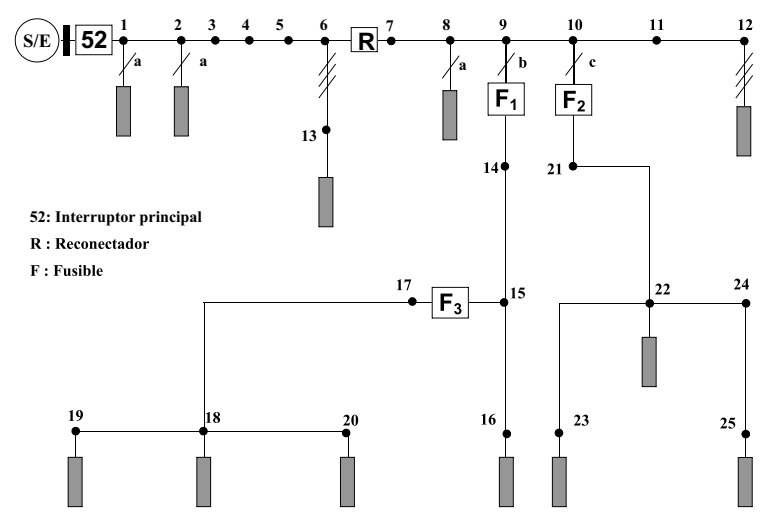

Figura 2. Sistema de distribución usado como prueba

\subsubsection{Modelado del sistema de distribución}

El proceso de incluir los elementos del sistema de potencia en la simulación es sencillo, gracias a la interfase gráfica del ATP conocida como ATPDraw (ATP, 2002). Se pueden seleccionar los diferentes elementos de una lista muy completa, la cual incluye generadores, líneas de transmisión (existen diferentes modelos de la línea), elementos pasivos, conmutadores, entre otros.

La simulación de las protecciones (las cuales no se encuentran dentro del listado mencionado anteriormente), se hace a través de los MODELS que posee el ATP. Éstos son una herramienta que permite crear nuevos elementos mediante una estructura de programación con un lenguaje de código de propósito general, soportado por un conjunto de herramientas de simulación para representación y estudio de las diferentes variables (ATP, 2002).

\subsubsection{Modelado del elemento de protección fusible}

Los fusibles son elementos que no necesitan dispositivos adicionales para su correcto funcionamiento como transformadores de corriente (CT's), y cumplen con la función de relé e interruptor. Son elementos que ofrecen protección, de acuerdo a una banda de característica inversa tiempo contra corriente. Ante una falla en el sistema de distribución, estos elementos actúan solamente una vez por expulsión o fundición (Lewis, 1998).
En la figura 3 se presenta el esquema del funcionamiento y la lógica de programación utilizada para simular la operación del fusible.

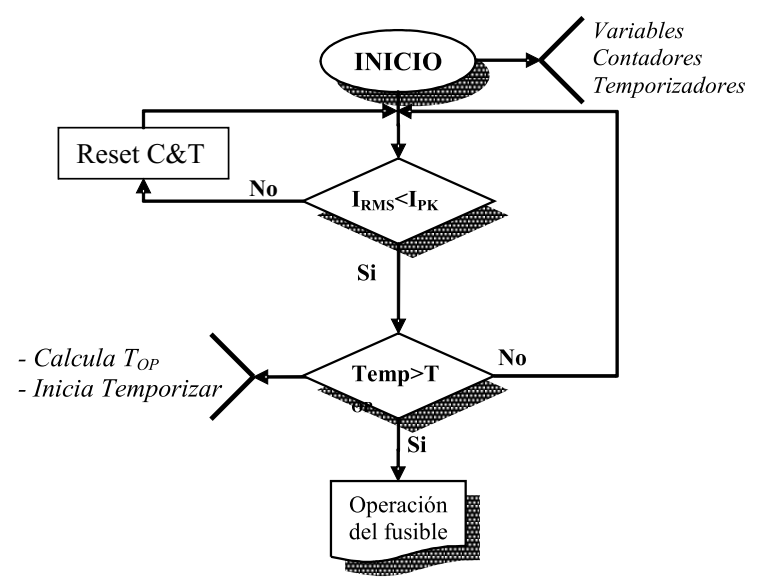

Figura 3. Esquema de funcionamiento del modelo de simulación del fusible

En la figura 4 se muestra el código de programación elaborado con MODELS del ATPDraw, para el modelamiento del fusible.

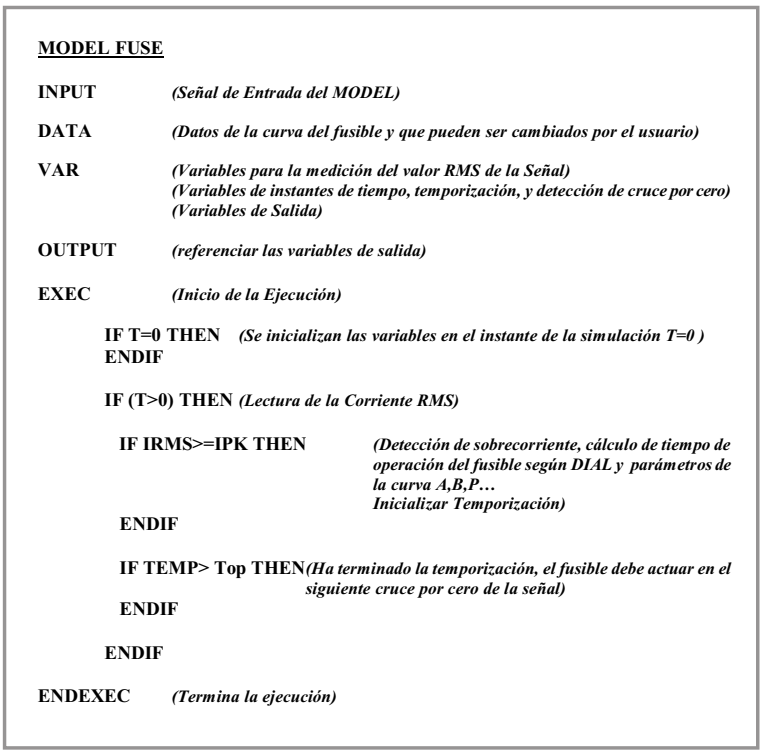

Figura 4. Ejemplo del código del MODEL del fusible

\subsubsection{Modelado del elemento de protección reconectador}

Los reconectadores cuentan con funciones de protección (50-51), interrupción (52), integradas con control de recierre del circuito. Estos 
elementos establecen una secuencia de recierre típica de las estructuras de automatización de la distribución.

Los reconectadores tienen comúnmente dos curvas, una rápida y una lenta. Ante una falla en el sistema de potencia, se estima la corriente de falla y actúa un número determinado de veces con la curva rápida y un tiempo de recierre tR. Si la falla persiste, el reconectador actúa un número determinados de veces con la curva lenta, recerrando el circuito con un tiempo tL. Estos elementos son normalmente utilizados en los sistemas de distribución para reducir el efecto de las fallas transitorias en el índice equivalente de interrupciones DES (Lewis, 1998).

El código implementado dentro del Model para la simulación de la operación real del reconectador, es muy similar al código del fusible (figura 4). El reconectador mide permanentemente el valor RMS de la corriente en cada una de las fases. En caso de existir sobrecorriente en el sistema, el reconectador inicia su proceso de operación, que consiste en operaciones rápidas y lentas (El reconectador fue programado con dos acciones rápidas y una lenta).

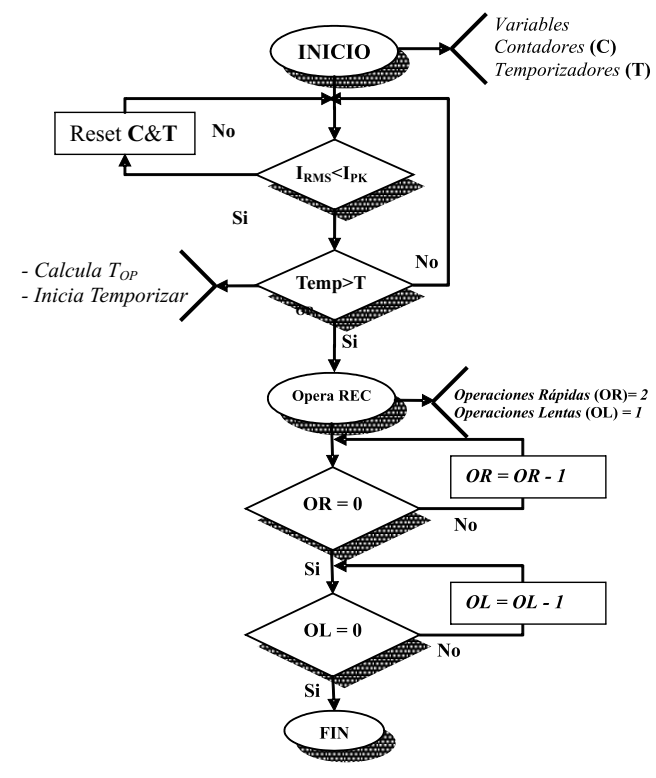

Figura 5. Esquema de funcionamiento del modelo de simulación del reconectador
Cuando hay fallas monofásicas, el reconectador tiene la posibilidad de hacer disparo monofásico. Para fallas bifásicas o trifásicas, el disparo es trifásico. Si durante la operación de apertura y recierre del reconectador, la corriente del sistema vuelve a la normalidad, éste inhibirá el conteo y ejecución de disparos.

La figura 5 muestra el funcionamiento y la lógica de programación del MODEL utilizado para simular la operación del reconectador.

\subsubsection{Simulación de sistemas de distribución en el ATPDraw}

La ejecución de las simulaciones en el ATPDraw se realiza de forma discreta. La simulación se inicia en el instante de tiempo cero y se realizan los cálculos respectivos para cada paso de simulación timestep; la simulación termina hasta llegar al tiempo final endtime; estos dos parámetros son fijados por el usuario.

El ATPDraw ejecutará el código programado de todos los Modelos que existan dentro del circuito en cada uno de los pasos de la simulación. La variable tiempo no se puede modificar por el usuario, sin embargo se puede acceder a la información del tiempo transcurrido de simulación dentro de los Modelos.

\subsection{Generación automática de fallas mediante ATPy Matlab®.}

Para la obtención de una base de datos con registros de tensión y corriente, se simulan diferentes tipos de fallas en el sistema de prueba de la figura 4. Esto es necesario ya que el conocimiento empírico necesita de una gran cantidad de ejemplos para el aprendizaje. La generación automática de fallas en ATP consiste en generar y simular automáticamente una gran cantidad de copias del sistema de distribución, sometido cada vez a una falla diferente.

El tipo de fallas simuladas son: Falla monofásica a tierra (Falla fase A tierra -Falla01, Falla fase B tierra - Falla02, Falla fase C tierra-Falla03), Falla Bifásica (Falla fases A y B. -Falla04, Falla fases B y C-Falla05, Falla fases A y C -Falla06), Falla Bifásica a tierra (Falla fases A y B y tierra. - A, B y 
Falla07, Falla fases B y C y tierra-Falla08, Falla fases A y C y tierra -Falla09), Falla Trifásica (Falla fases A, B y C -Falla10; Falla fases A, B y C y tierra -Falla11).

Para tener una base de datos completa, estas fallas se simularon en cada uno de los nodos del sistema (Considerando que existen ramales monofásicos en los cuales sólo se puede simular un solo tipo de falla). Además, cada una de las fallas, se simuló con seis resistencias de fallas diferentes que son: $0.5,5,10,20,30,40$.

La interacción entre ATP y Matlab para la simulación automática de fallas es mostrada en la figura 6 , y se describe a continuación:

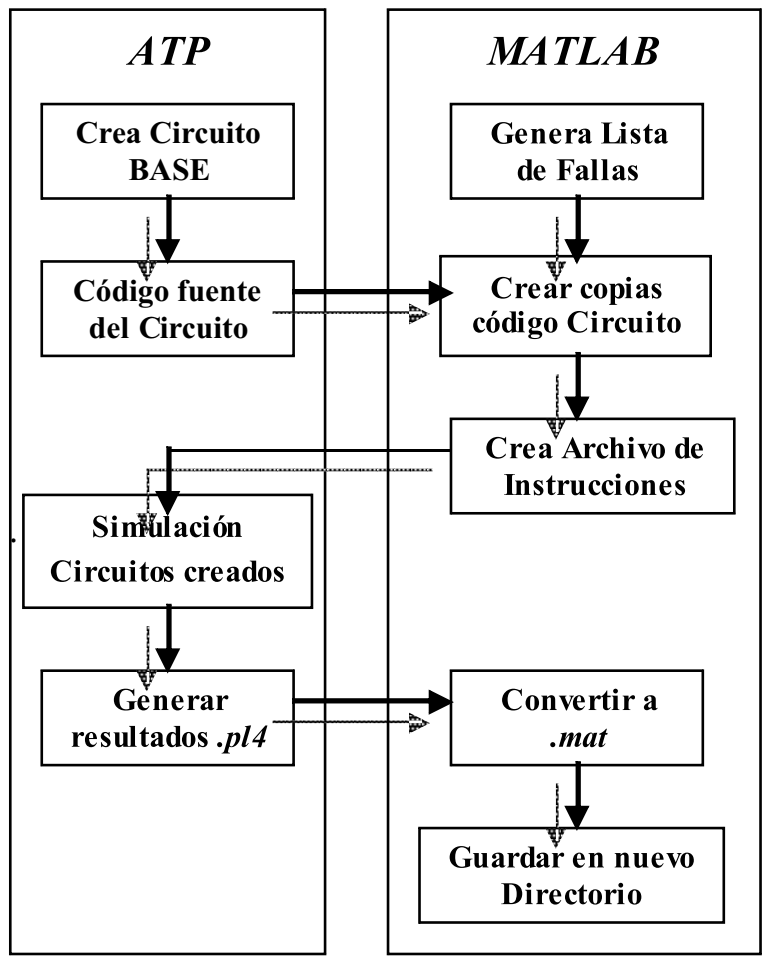

Figura 6. Esquema de interacción implementada entre ATP y Matlab para la generación automática de fallas

\subsubsection{Generación de un listado de fallas}

Para la generación del listado de fallas se realizó un programa con la ayuda del software Matlab®. Esta aplicación se encarga de generar un archivo de texto (con extensión .txt), con un listado de fallas. La forma de generar este listado, es mediante la generación y concatenación de texto y números convertidos a caracteres.

El nombre de cada archivo ATP contenido en el archivo de texto es "FallaXXBusYYRFZZ.atp", donde XX corresponde al tipo de falla. (De la 01 a la 11), YY es el nodo donde se simula la falla (Del 01 a 25 nodos del sistema de prueba), y ZZ es el valor de la resistencia de falla (Numerados de $01 \mathrm{a}$ 06).

\subsubsection{Generación de los diferentes circuitos en el ATP}

Este proceso se realiza a partir de un archivo base, el cual tiene el código ATP del sistema de potencia dibujado en el ATPDraw y complementado con las protecciones desarrolladas en los Modelos. Basta con modificar algunas líneas del código del archivo para cambiar el valor de las resistencias de falla y la ubicación del nodo de falla. En esta etapa se genera un gran número de copias del mismo sistema de distribución pero cada uno tiene asociada una falla diferente.

\subsubsection{Generación del archivo de ejecución automática de los archivos ATP de fallas}

Para la ejecución automática de los circuitos generados anteriormente, se utiliza un archivo de instrucciones de procesamiento por lotes (batch) de extensión .bat que ejecuta todas las simulaciones. Las instrucciones que contiene este archivo son:

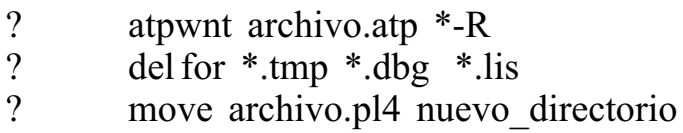

La primera instrucción se encarga de ejecutar los archivos .atp generados en la etapa anterior. En la simulación de un archivo .atp se generan otros archivos de extensión .lis .dbg, .tmp y .pl4 de los cuales sólo interesa este último, de modo que la segunda instrucción se encargará de borrar los demás archivos. Finalmente, la tercera instrucción se encarga de mover los archivos .pl4 a un nuevo directorio. Éstos últimos corresponden a los resultados de la simulación de los archivos .atp. 
Para este caso y acorde con los datos que se disponen en los sistemas de distribución reales, la base de datos de registros de fallas queda conformada por las señales de tensión y corriente en el dominio del tiempo y sus valores RMS, medidos en el nodo de la subestación.

\subsubsection{Conversión de los resultados de los archivos.pl4 a archivos .mat}

Los archivos de extensión .mat son archivos de datos en código ASCII, que pueden ser manipulados por el Matlab. En este archivo se guardan las señales de tensión y corriente en el dominio del tiempo y sus valores RMS. Teniendo en cuenta la potencia de manipulación de datos de Matlab ${ }^{\circledR}$, se creó un archivo que se encarga de convertir los archivos .p14 en archivos .mat.

Una vez se tienen los resultados como archivos de extensión .mat se puede realizar con el software Matlab los respectivos análisis y procesar las señales de tensión y corriente obtenidas del ATP.

\subsubsection{Obtención de descriptores}

Mediante el procesamiento de los registros de tensión y corriente de falla, se obtienen los descriptores definidos en el numeral III $\mathrm{C}$ y que servirán para entrenar y validar los $\mathrm{MBC}$ elaborados.

\section{Resultados y discusión}

El sistema de pruebas contiene 25 nodos; 13 de ellos son nodos trifásicos y se simularon 11 tipos de fallas con seis valores diferentes de resistencia de falla, lo cual da como resultado 858 simulaciones. Los 12 nodos restantes son monofásicos y en ellos se simuló un solo tipo de falla con seis valores de resistencia diferentes, lo cual da como resultado 72 simulaciones.

De cada una de las simulaciones se extrajeron las señales, en el dominio del tiempo, de tensión y corriente medidas en el nodo de la subestación. Además se extrajeron los valores RMS de dicha señal. La base de datos para este sistema, almacenando únicamente los archivos .mat tiene un tamaño de 1.04 GB. El tamaño de la base de datos es relativamente grande, esto se debe a la frecuencia de muestreo de la señal $(20 \mathrm{kHz})$ y el tiempo total de simulación de la falla $(1.1 \mathrm{~s})$.

En total se simularon 930 casos de fallas diferentes y de forma automática, con una duración total del proceso de 36 horas. Hacer el mismo número de simulaciones manualmente no sería práctico, pues se requiere de días de trabajo en los cuales debe estar presente permanentemente el operador.

A manera de ejemplo, se muestran los descriptores del sistema obtenidos para una falla trifásica en el nodo 6 con una resistencia de $40 \Omega$. Las frecuencias obtenidas para el transitorio son 3740 $\mathrm{Hz}, 3780 \mathrm{~Hz}$ y $3760 \mathrm{~Hz}$; y las duraciones del transitorio son $8.05 \mathrm{~ms}, 8.35 \mathrm{~ms}$ y $9.35 \mathrm{~ms}$, para las fases $\mathrm{A}, \mathrm{By} \mathrm{C}$, respectivamente.

En cuanto al descriptor de los patrones de la corriente de carga, se obtuvieron los tiempos de detección de fallas y la operación de las distintas protecciones así: [40 - 393 - 593 - 948] ms. Al nodo seis sólo está asociada la acción del interruptor principal de la cabecera del circuito. El vector anterior indica que a los $40 \mathrm{~ms}$ de simulación ocurrió la falla, $353 \mathrm{~ms}$ después el interruptor principal la libera, $200 \mathrm{~ms}$ después el interruptor hace un recierre, pero como la falla es permanente, $355 \mathrm{~ms}$ después el interruptor principal abre de nuevo el circuito, permanentemente hasta el final de la simulación $(1100 \mathrm{~ms})$.

Actualmente, los resultados obtenidos en este trabajo están siendo usados para resolver el problema de localización de fallas mediante Máquinas de Soporte Vectorial y el Método LAMDA.

\section{Conclusiones}

La calidad de la energía es un tema de vital importancia para las empresas de distribución. La continuidad en el suministro es uno de los aspectos más importantes para los usuarios debido al impacto económico y social generado a partir de la ocurrencia de interrupciones, y para las empresas debido a las implicaciones económicas en las que incurren en caso de una deficiente prestación del servicio. 
La simulación del esquema de protección utilizado en los sistemas de distribución, mostrado en este trabajo, refleja un comportamiento real de los circuitos y permite extraer información importante (descriptores), que es requerida por las metodologías basadas en el conocimiento para resolver el problema de localización de fallas.

Se han implementado, por medio de herramientas ampliamente reconocidas por los investigadores a nivel mundial (ATP y Matlab), modelos para protecciones de sistemas de potencia como fusibles y reconectadores. Estos modelos pueden ser útiles para diferentes aplicaciones que constantemente involucran dichos elementos.

La simulación automática de fallas ha permitido crear una gran base de datos que describe todo el sistema de distribución bajo cualquier evento. Realizar el mismo número de simulaciones de forma manual no sería práctico.

Este trabajo es la base para la solución del problema de localización de fallas en sistemas de distribución, ya que de forma automática se ha obtenido la simulación de un sistema de distribución bajo todas las posibles condiciones de falla. Esta información es utilizada no sólo por los métodos basados en el conocimiento (MBC) sino también por las metodologías basadas en el modelo de la red (MBM), necesarias para resolver el problema de localización.

\section{Referencias bibliográficas}

ATP (2002). Alternative Transient Program Rulebook. Comité Argentino de Usuarios del EMTP/ATP-CAUE.

Burges, C.J.C. (1998). A tutorial on support vector machines for patter recognition. Data Mining and Knowledge Discovery 2(2), 121-167.

Carrillo, G., \& Ordóñez, G. (2002). Calidad del Suministro de Energía Eléctrica (Power Quality). Tutorial Course, ALURE Program-CREG Project, Colombia.
Cormane, J., Vargas, H., \& Ordóñez, G. (2005). Modelo Estadístico para la Localización de Fallas en Sistemas de Distribución. III Simposio Internacional sobre Calidad de la Energía Eléctrica SICEL 2005, Bogotá, Colombia.

CREG 1998-2002. Resoluciones CREG 070 de 1998, CREG 096 de 2000, CREG 084 de 2002, CREG 084 de 2002. Comisión de Regulación de Energía y Gas CREG.

Das, R. (1998). Determining the Locations of Faults in Distribution Systems. Doctoral Thesis, University of Saskatchewan, Saskatoon, Canada.

Lewis, J. (1998). Protective relaying principles and applications. Marcel Dekker, Inc.

Li-Xing, W. (1997). A course in fuzzy systems and control. Prentice Hall, Inc.

Martínez, J., \& Castro, J. (2002). Análisis de la teoría de ondículas orientada a las aplicaciones en ingeniería eléctrica: Fundamentos. Departamento de Ingeniería Eléctrica, E.T.S. Industriales, Madrid.

Martins, L.S., Martins, J.F., Alegria, C.M., Fernão-Pires, V. (2003). A network distribution power system fault location based on neural eigenvalue algorithm. IEEE Bologna PowerTech Conference.

Mora, J. (2003). Voltage sag characterization and classification for diagnosis in electric power quality domain. Master Thesis. University of Girona, Spain.

Mora, J., Muñoz, S., \& Carrillo, C. (2005a). Técnicas algoritmicas de localización de fallas como alternativa para reducir el efecto de las salidas en sistemas de potencia - Una revisión. III Simposio Internacional sobre Calidad de la Energía Eléctrica SICEL 2005, Bogotá, Colombia. 
Mora, J., Meléndez, J., \& Carrillo, G. (2005b). Una arquitectura genérica para el desarrollo de sistemas hibridos. propuesta de localizador de fallas para mejorar los indices de calidad. III Simposio Internacional sobre Calidad de la Energía Eléctrica SICEL 2005, Bogotá, Colombia.

Mora, J., Serrano, H., \& Ordóñez, G. (2005c). Mejoramiento de los índices de calidad del servicio mediante la caracterización de señales para localización de fallas en distribución. III Simposio Internacional sobre Calidad de la Energía Eléctrica SICEL 2005, Bogotá, Colombia.

Phadke, A., \& Horowitz, S. (1995). Power system relaying. Baldock, England: Research Studies Press.
Russell, S., \& Norvig, P. (2002). Artificial intelligence: a modern approach. Second Edition, Prentice Hall.

Seung-Jae, L., Myeon-Song, C., Sang-Hee, K., Bo-Gun, J., Duck-Su, L., Bok-Shin, A., NamSeon, Y., Ho-Yong, K., \& Sang-Bong, W. (2004). An intelligent and efficient fault location and diagnosis scheme for radial distribution systems. IEEE Transactions on Power Delivery 19 (2), $524-532$.

Torres, H., Acero, G., Flechas, J., Saucedo, J., \& Quintana, C. (2001). Curso de calidad de la energía eléctrica CEL. ACIEM, Cundinamarca.

Waissman, J., Aguilar, M., \& Dahhou, B. (2000). Construction d'un modele comportemental pour la supervision de procedes: application a une station de traitement des eaux. (Cap. 2). Doctoral Thèse, Institut National Polytechnique de Toulouse. France. 